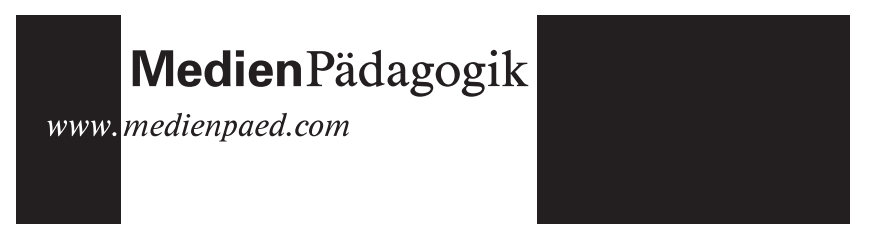

Carsten Schulte

4.12 .2001

\section{Informatische Aufgabenbereiche der Medienbildung}

\section{Informatik und Medien}

In den neunziger Jahren wurden die Möglichkeiten und Auswirkungen der Informationstechnologie in Medienzusammenhängen verstärkt deutlich. Das Internet wurde als Motor für einen grundlegenden Wandel in der Gesellschaft gesehen: Die Informationsgesellschaft versprach freien Zugang zur Informationen für alle, einen schonenden und unaufhaltsamen Wirtschaftsaufstieg, das Zusammenwachsen der Nationen und Kulturen. Die Schulen gingen ans Netz. Neue Medien bedeuteten neue Aufgaben und Anforderungen:

- eine Informationskompetenz als die Fähigkeit, aus grossen Datenmengen die benötigten herauszufiltern, was ohne Werkzeugeinsatz nicht mehr möglich erschien;

- eine Lesekompetenz, die noch stärker die Frage nach der Authentizität der übermittelten Informationen im Blick hatte;

- eine Gestaltungskompetenz, um die Daten auch multimedial aufzubereiten und im Netz bereitzustellen;

- eine grössere soziale Kompetenz, um die zunehmende Entfernung und Anonymisierung zwischen Kommunikationspartnern im Netz aufzufangen [vgl. PSW98].

Diese Aspekte wurden den bereits bestehenden Medienbildungsaufgaben der Schule additiv hinzugefügt. Computerspiele, Multimedia und die Kommunikation in Netzen waren zusätzliche Bereiche für die medienerzieherische Arbeit. Neu war die Perspektive, dabei den Computer als Werkzeug zur Gestaltung von Medien einzusetzen.

Die Aufgabenbereiche der Medienerziehung sollten sowohl für die 〈alten〉, als auch für die «neuen〉 Medien gelten: Medieneinflüsse erkennen und auf- arbeiten, Medienbotschaften verstehen und bewerten, Medienangebote auswählen, Medien gestalten, die gesellschaftliche Bedeutung der Medien analysieren und beeinflussen [HT96]. Die Medienbildung liess sich damit unabhängig von den technischen Besonderheiten einzelner Medien in einem übergreifenden Konzept darstellen, das aber um den Preis einer sehr allgemeinen Sichtweise.

In den computerbasierten Medien wie WWW, Multimedia, E-Mail, etc. nehmen die technischen Aspekte eine besondere Rolle ein. Diese Rolle ist für die Medienbildung genauer herauszuarbeiten. Den vielfältigen technischen Realisierungen liegt eine begrenzte Zahl informatischer Grundprinzipien zugrunde, mit denen die Spezifika computerbasierter Medien im Wesentlichen erklärt werden können. Zu diesem Zweck werden dann informatische Aufgabenbereiche ${ }^{1}$ der Medienbildung bestimmt.

\section{Computerbasierte Medien aus der Sicht der Informatik}

In der Informatik, insbesondere in der Teildisziplin «Informatik und Gesellschaft>, wird zunehmend auch die mediale Komponente der Informatik und des Computereinsatzes diskutiert. Dies erfolgt beispielsweise mit Hilfe semiotischer Sichtweisen.

\section{Informatik und Semiotik}

In der semiotischen Perspektive erscheint «der Computer [..] als Medium, weil er an zwei Arten von Zeichenprozessen teilhat. [..] Darin liegt das Besondere des informatischen Zeichens - in seiner Eigenschaft, gleichzeitig Signal für die Maschine und Zeichen für den Menschen sein zu können» [vgl. Wi00].

Dieser Zusammenhang ist eine notwendige Voraussetzung für das Verstehen der spezifischen medialen Funktionen des Computers [vgl. Ke00]: Die Möglichkeit der automatisierten Verarbeitung von Zeichen wird nicht nur für die Erstellung, Speicherung und Übertragung von Zeichen genutzt. Ihre Anwendung zur Transformation kann an verschiedenen Punkten der Kette Autor - Nachricht - Empfänger ansetzen. Ein Beispiel hierfür ist das

${ }^{1}$ Der Begriff der informatischen Aufgabenbereiche soll zum einen nahelegen, dass es lediglich um einen Teil des Medienspektrums, nämlich um computerbasierte oder auch informatische Medien handelt. Zum anderen soll die Bedeutsamkeit informatischer Bildung als notwendiger Bestandteil einer Medienbildung in Abgrenzung zu eventuell schnell veraltenden Kenntnissen in der Bedienung spezieller Hard- und Softwareprodukte verdeutlicht werden. 
ursprüngliche Konzept der Auszeichnungssprache html: Nicht der Autor sondern der Empfänger der html-Seite bestimmt mit Hilfe seines Browsers das Aussehen der Seite. Dies war (Anfang der 90er Jahre) eine wichtige Funktion, um Informationen über Rechner- und Betriebssystemgrenzen hinweg austauschen zu können. Einfache Displays zur Darstellung weniger Textzeilen konnten für die Ausgabe ebenso eingesetzt werden wie die ersten Farbmonitore. Aus semiotischer Perspektive geriet damit ein Fixum schriftsprachlicher Zeichensysteme ins Wanken: Schriftzeichen wurden und werden als Markierungen auf einem Beschreibstoff, sei es Papier oder die Phosphorschicht des Monitors, definiert. Hier jedoch findet eine Aufspaltung der Zeichen in Codes und Fonts statt, so dass algorithmische Zeichentransformationsprozesse denselben Text unterschiedlich darstellen können [We95].

Auf dieser Trennung baut eine weitere Ebene automatisierter Zeichentransformationen auf: die Möglichkeit, Nachrichten oder andere Medienangebote automatisiert oder teilautomatisiert zu erzeugen. Als Beispiel sei der Internet-Auftritt grosser Versandhäuser genannt, die ihren Kunden beim erneuten Aufruf der Webseite automatisch individuelle Angebote auswählen und präsentieren, die sie anhand früherer Bestelldaten oder erstellter Kundenprofile zusammengestellt haben.

Als weiteres Beispiel für die automatisierte Zeichentransformation dient Maurers Beschreibung eines Werkzeugs zur Erhöhung der Interaktivität von Medienangeboten im WWW. Die Unterstützung eines Autors (facilitator) bei der Entwicklung eines interaktiven Medienangebots durch ein solches «collaborative tool» (ct) kann wie folgt aussehen: «Such a ct might provide a number of further features for the facilitator that come in handy: a toggle to send a <thanks for your contribution〉 message automatically, statistical information of how often a pen name has contributed over certain periods causing a <thanks for your many contributions〉 message or 〈hope you are not sick, because you have not contributed for four weeks $>$ message, etc.: such and other messages can be configured by the facilitator [..] In most cases such mails should not be sent entirely automatically, but through the «hands of the facilitator to allow to add personal touch.» [Ma99].

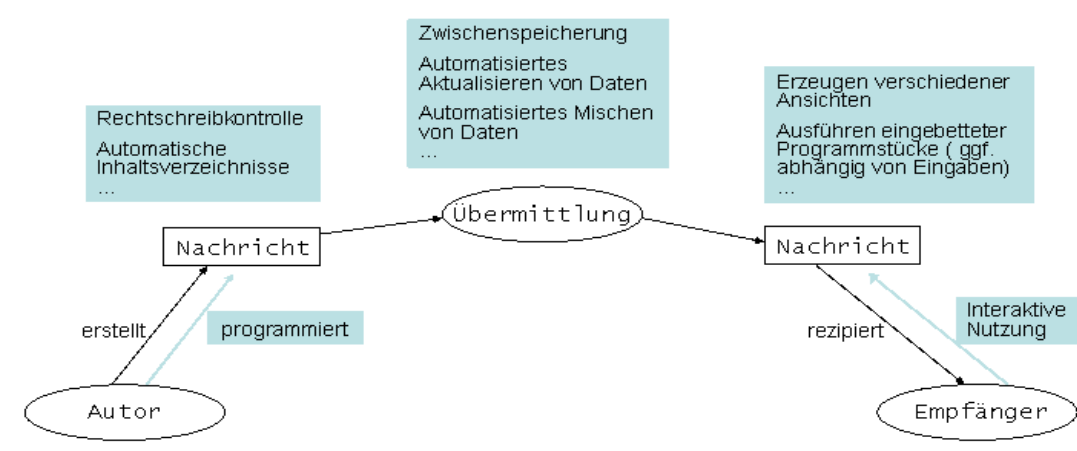

Abb. 1: Spezifische Möglichkeiten von computerbasierten Medien zur Erstellung von Medienangeboten

Aus der Perspektive der Anwender werden computerbasierte Medien unter anderem als «Ausdrucksmittel des Geistes» [Ke00] dargestellt, d. h. sie fungieren als Werkzeuge, um Repräsentationen herzustellen. Diese Repräsentationen sind Arrangements von Zeichen, die mit Hilfe computerbasierter Medien erzeugt und gepflegt werden können. Technische Funktionen unterstützen somit geistige Vorgänge und werden als Medienfunktionen dargestellt: «Die Möglichkeiten, mit Hilfe von Technik solche Arrangements [von Zeichen] aufzubauen und zu pflegen, indem Zeichen und Artefakte erzeugt, ins Wahrnehmungsfeld gebracht und arrangiert sowie dauerhaft verknüpft werden können, bilden die primären Medienfunktionen:

1.Erzeugen: «Ein Phänomen wahrnehmbar machen [..] (schreiben, simulieren, messen, visualisieren)»

2.Arrangieren: «Verschiedene Artefakte möglichst gleichzeitig ins Wahrnehmungsfeld bringen und so anordnen, dass inhaltliche Zusammenhänge räumlich abgebildet werden können (strukturieren, recherchieren, ordnen, vergleichen)»

3.Verknüpfen: Zusammengehörige Artefakte als eine Einheit behandeln [.. durch physisches Verbinden, Zusammenfassen in einen Behälter oder durch das Anlegen von Verweisen» [Ke00]. 
Primäre Medienfunktionen werden bereits bei der Nutzung einer Textverarbeitung eingesetzt. Wenn aus dem Text ein interaktives Web-Angebot erstellt wird, kommen automatisierte Transformationsprozesse hinzu. Zusätzliche technische Anforderungen treten auf, falls der Autor Interaktionsmöglichkeiten programmiert oder für die interaktive Nutzung technisches Hintergrundwissen benötigt wird.

\section{Informatik und soziale Strukturen}

In der Teildisziplin Informatik und Gesellschaft wird die besondere Rückbezüglichkeit informationstechnischer Artefakte erforscht: die Wechselwirkungen zwischen Informatik und Gesellschaft. Als zweckintentionale Artefakte werden sie in den Handlungssituationen verwendet für die sie gestaltet worden sind. Doch gerade durch diesen Einsatz ändert sich die ursprüngliche Handlungssituation und das Artefakt ist demzufolge nicht passgenau für seine Einsatzumgebung gestaltet, der Wunsch nach Änderungen nahezu unvermeidlich [Le80]. Damit kommt ein Gestaltungsprozess in Bewegung, der über die Entwicklung eines einzelnen Artefakts hinausweist und eine technisierte Umwelt entstehen lässt, die leicht mit einer natürlichen Umwelt verwechselt wird.

Lawrence Lessig bemerkt dazu: «Too many miss how different architectures embed different values and that only by selecting these different architectures - these different codes - can we establish and promote our values» [Le99]. Im Code, dem Quelltext der Software, bzw. in der Architektur unserer technischen Umwelt sind implizit Wertentscheidungen enthalten, die prinzipiell änderbar sind und durch Veränderung der Architektur - des Quelltextes - auch geändert werden können. Lessig nennt als Beispiel Eigenschaftszuschreibungen an das Internet, die das weltweite Netz als unregulierbar, frei, offen und prinzipiell zugänglich für jeden darstellen. Diese Beschreibungen suggerieren, sie könnten das Internet an sich〉 erfassen - tatsächlich werden die Eigenschaften einer bestimmten Softwaregeneration des Internet beschrieben.

Diese Eigenschafts-Beschreibungen des Internet verdeutlichen jedoch, dass implizit in technischen Realisierungen Werturteile enthalten sind, hier etwa die Idee des freien, unregulierbaren Zugangs zu allen im Internet verfügbaren Informationen. Diese Werturteile können sich allerdings ändern. So wird mittlerweile die Rolle des Urheberschutzes stärker betont, was sehr wahrscheinlich dazu führen wird, dass auch die Architektur des Netzes geändert wird, um die neuen Werturteile zu implementieren〉.
Keil-Slawik beschreibt diesen Aspekt aus einer medialen Sichtweise: Software transformiert Informationen mit Hilfe algorithmischer Verfahren nach festen Regeln. Der Einsatz von Software erfolgt in den meisten Fällen über interaktive Benutzungsschnittstellen. Durch interaktive Nutzung ist der Transformationsprozess kein in sich geschlossener Ablauf, sondern eingebettet in soziale Strukturen der Nutzung, in welchen den verarbeiteten Zeichen mit Hilfe sozialer Konventionen Bedeutung zugewiesen wird. Damit sind in den Softwarestrukturen zumindest implizit menschliche Verhaltensweisen, Werte und Normen abgebildet. Durch den Einsatz von Software an einem Arbeitsplatz ändern sich beispielsweise die zugeordneten Aufgaben, was auch wieder auf das Verhalten der Menschen zurück wirkt. Solche Änderungen führen in vielen Fällen wiederum zu Änderungen an der Software, etwa um die darin enthalten impliziten Modelle menschlichen Verhaltens an die veränderte Situation oder an neue gesetzliche Regelungen anzupassen.

\section{Neuere Ansätze der Informatikdidaktik}

Ursprünglich zielte die informatische Bildung nahezu ausschliesslich auf Programmierfähigkeiten und -kenntnisse. In den 70er, 80er und bis in die 90er Jahre hinein galt das algorithmische (bzw. informatische) Problemlösen als die Fähigkeit, Probleme aus der Alltagswelt mit Hilfe der Informatik formal zu beschreiben, eine algorithmische Lösung zu finden, ausrechnen zu lassen und die Ergebnisse reflektiert nutzen zu können. Diese Form des Problemlösens kann in etwa mit Programmieren gleichgesetzt werden [Sc01a]. Doch die oben beschriebenen Sichtweisen finden zunehmend Eingang in didaktische Ansätze für den Informatikunterricht, so dass Computer auch als Medien, die Computernutzung auch als Mediennutzung gesehen werden und informatische Bildung nicht mehr auf Programmierfähigkeiten beschränkt bleibt. Die beiden folgenden informatikdidaktischen Ansätze zielen auf Nutzungskompetenzen im Umgang mit interaktiven Systemen, die als Sonderfälle computerbasierter Medien angesehen werden können.

Der informationszentrierte Ansatz der Informatikdidaktik

In ihrem informationszentrierten Ansatz gehen Hubwieser und Broy davon aus, dass angesichts einer immer grösser werdenden Informationsmenge das Umgehen mit Informationen ohne computerbasierte Hilfsmittel nicht mehr möglich ist [HB97]. Das Strukturieren, Aufbereiten, Finden und Ver- 
walten von Informationen wird zur Leitlinie des Informatikunterrichts. Der Begriff Information wird als (quantifizierbare) Datenmenge verstanden. Im Mittelpunkt des informationszentrierten Ansatzes stehen Notationen und grafische Beschreibungssprachen für Informationsmengen.

Für die unterrichtliche Umsetzung wird empfohlen, beginnend mit der sechsten Jahrgangsstufe, Erstellung, Präsentation, Verwaltung, Transport und automatisierte Verarbeitung von Dokumenten verschiedenen Typs (Text, Grafik, Tabellen) zu thematisieren. Dem schliesst sich in der Sekundarstufe II als vertiefte informatische Bildung die Repräsentation von Informationen, die Datenmodellierung, die zustandsorientierte, die funktionale und die objektorientierte Modellierung an, ergänzt durch eine Unterrichtseinheit über Softwareprojekte [Hu00].

Dabei ist wichtig, dass die im Unterricht vermittelten informatischen Modellierungssprachen auch ausserhalb der Softwareentwicklung zur Beschreibung von Sachverhalten eingesetzt werden können ${ }^{2}$.

Am Beispiel aktueller Softwarepakete und Notationen werden somit statt Bedienkenntnisse informatische Beschreibungstechniken vermittelt - formal-logische Kenntnisse, welche die Grundlage für eine lebenslang neu zu erarbeitende und zu aktualisierende Nutzungskompetenz bilden können. Hubwieser [Hu00] nennt als Einführungsbeispiel für die Sekundarstufe I die Planung einer Wohnungseinrichtung unter Nutzung eines Vektorgrafikprogramms. Die Einrichtung wird aus der Vogelperspektive dargestellt. Durch Verschieben der Elemente wird die Einrichtung geplant. Im Zusammenhang mit der Nutzung des Programms wird das zugrunde liegende Datenmodell entwickelt. Andere Einheiten thematisieren Aufbau und Versand von E-Mails, Hypertexte und Übertragungsprotokolle [Hu00].

Der systemorientierte Ansatz der Informatikdidaktik

Während der informationszentrierte Ansatz auf die Kompetenz des Einzelnen in einer (von Software) vorgegebenen 〈Umwelt〉 fokussiert, will die

\footnotetext{
2 Sachverhalte beziehen sich in diesem Zusammenhang auf zwei unterschiedliche Aspekte: Zum einen kann man mit Software-Werkzeugen beliebige Inhalte darstellen, wie Texte, Grafiken, Bilder. Insofern beziehen sich Sachverhalte auf Darstellungen, die mit Computer-Programmen erzeugt (ausgedrückt) werden. Zum anderen werden 〈Formale Beschreibungsverfahren der Informatik〉 vermittelt, etwa zur Darstellung automatisierter E-Mail-Antworten oder um formale Strukturen (z. B. Schulorganisation) darzustellen. Sachverhalte sind somit auch formale Strukturen, die mit informatischen Notationen ausgedrückt werden.
}

systemorientierte Didaktik vor allem das Künstliche, das Geschaffen-Sein informationstechnischer 〈Umwelten〉 vermitteln und auf die stets notwendige Zweckbindung technischer Produkte hinweisen.

Kernelement dieser Betrachtungsweise ist das soziotechnische Informatiksystem: Die technischen Komponenten werden zusammen mit und eingebettet in soziale Handlungssysteme thematisiert, um zu verdeutlichen, dass die Gestaltung und Herstellung von Informatiksystemen auf einen erhofften Nutzen aus seinem Gebrauch zielt. Entweder wird das Artefakt von Menschen benutzt, oder die Ergebnisse der Funktionsweise dienen der Vollbringung der Arbeit von Menschen, sollen sie unterhalten, schützen, überwachen, etc.

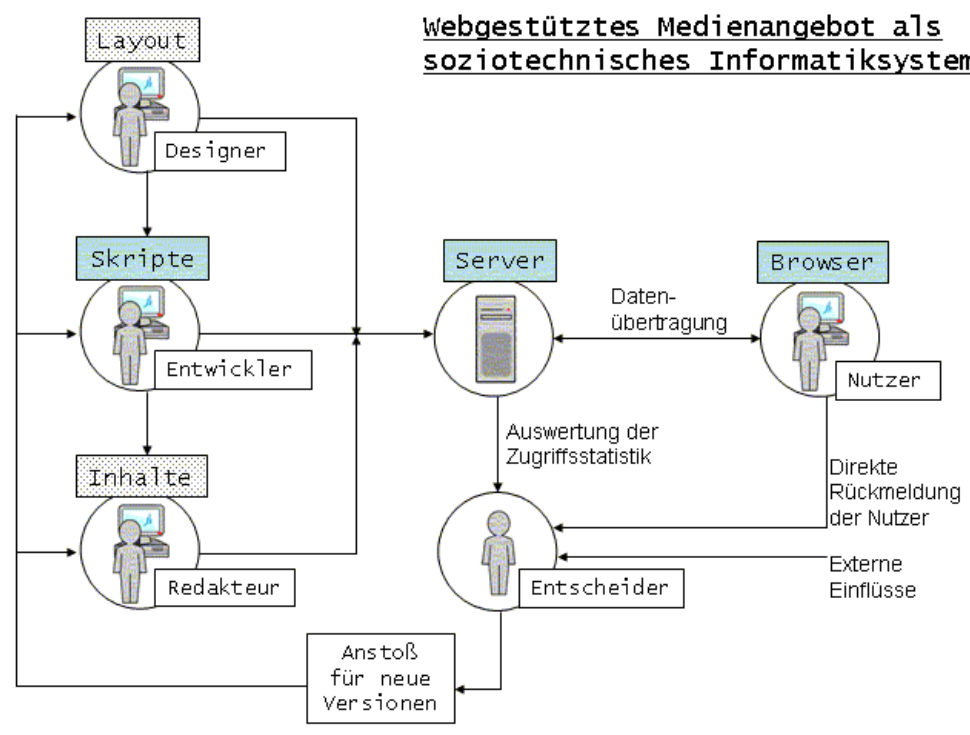

Abb. 2: Ein webgestütztes Medienangebot als soziotechnisches Informatiksystem nach [Sc01b].

Schulte und Scheel stellen ein Quiz im WWW aus der systemorientierten Perspektive dar. Im Unterricht werden nicht nur die Nutzung des Quiz (Relation: Nutzer-Server) und Programmierung des Quiz (Relation: Entwickler-Server), sondern auch begleitende Prozesse - vor allem der Gestaltungsprozess, der zu neuen Versionen der Software führt - angesprochen. Es wird verdeutlicht, dass ein solches Webangebot nicht allein aufgrund der grafischen Nutzungsschnittstelle beurteilt werden kann: In dem vorge- 
stellten Beispiel werden die bei der interaktiven Nutzung erzeugten Verbindungsdaten gesammelt und auf dem Server gespeichert. Implizit wird in dieser Darstellung auch deutlich, dass inhaltliche, ästhetischgestalterische und technische Anforderungen beim Erstellen eines webgestützten Medienangebots zu beachten sind.

\section{Vier Aufgabenbereiche informatischer Medienbildung}

Die im Folgenden zu entwickelnden informatischen Aufgabenbereiche sollen die notwendigen informatischen Elemente einer Medienbildung beschreiben, mit denen die computerbasierten Medien in angemessener Form berücksichtigt werden. Zu diesem Zweck werden zunächst bestehende Defizite weiter konkretisiert.

Die Funktion von Medien als Ausdrucksmittel für die Repräsentation von Inhalten mit Hilfe technischer Medien spielt bislang in der Medienerziehung keine Rolle. Durch die interaktiven Möglichkeiten computerbasierter Medienangebote, durch die spezifischen Ausdrucks- und Kommunikationsmöglichkeiten alltäglicher Softwareprodukte wie E-Mail, Textund Bildverarbeitung, Tabellenkalkulation gewinnt dieser Aspekt jedoch an Bedeutung.

Die Aufgabe, die Arbeitsweise bzw. die Wirkprinzipien eines Mediums zu verstehen, erweist sich bei computerbasierten Medien als besonders schwierig: ihre technischen Funktionsprinzipien werden vor dem Nutzer verborgen und die Interaktion mit dem Medienangebot, dem SoftwareWerkzeug, erfolgt über eine eigens gestaltete Benutzungsschnittstelle. Klaus Brunnstein beschreibt die Missverständnisse, die durch das WYSIWYG-Prinzip (What You See Is What You Get) hervorgerufen werden: «Zwar wird durch graphische Oberflächen der Eindruck erweckt, der Benutzer (besser: Bediener) habe den vollen Durchblick, da er alles Wesentliche für sein Ergebnis 〈sehe〉 [..].» Tatsächlich aber sind selbst in einfachen lokalen (nicht-vernetzten) Systemen die Schichten [ineinandergreifender Softwarebausteine und Hardwarekomponenten] so komplex [..] dass kein Mensch in der Lage ist nachzuvollziehen, ob das Erreichte auch das erwünschte oder das spezifizierte Ergebnis ist» [Br01].

Das Bewusstsein dieser Trennung von Ein-/ Ausgabenfunktionen auf der Oberfläche und den internen Wirkprinzipien gehört zu den notwendigen Voraussetzungen für eine kompetente Nutzung computerbasierter Medien.
Vor dem Hintergrund der informatikdidaktischen Ansätze und der Diskussion in der Informatik lassen sich vier Aufgabenbereiche für eine informatische Medienbildung beschreiben [vgl. Sc01b]:

\section{Der ausdrucksbezogene Aufgabenbereich}

Die Informatik nutzt und entwickelt Notationen zur Darstellung von Informationen, die auch ausserhalb von Softwareentwicklungsprozessen einsetzbar sind. Ziel des ausdrucksbezogenen Aufgabenbereichs ist es, das notwendige informatische Hintergrundwissen und das Handwerkszeug zu vermitteln, um diese Beschreibungstechniken kompetent zu nutzen, d.h. um mit Werkzeugen der Informatik Sachverhalte ausdrücken zu können. Diese informatischen Beschreibungstechniken lassen sich aus Eigenschaften von Datenformaten, Dateiablagestrukturen, medientypischen Werkzeugfunktionen, Notationen (vgl. Hubwieser) und aus den primären Medienfunktionen Erzeugen, Arrangieren, Verknüpfen, (vgl. Keil-Slawik) ableiten.

Ausdrucksbezogen bezieht sich in diesem informatischen Aufgabenbereich allerdings nicht auf inhaltliche und ästhetische Fragen der Gestaltung von Medienangeboten.

\section{Der Aufgabenbereich technische Grundlagen}

Dieser Bereich beschreibt Voraussetzungen an technischem und informatischem Wissen für eine informatische Medienbildung. Die Inhalte dieses Aufgabenbereichs ergeben sich implizit aus den Anforderungen der anderen Bereiche. Um mit Werkzeugen der Informatik etwas auszudrücken, werden technische Hintergrund-Kenntnisse benötigt, etwa um passende Datenformate auszuwählen, Dateiablagestrukturen zu gestalten, Werkzeuge auszuwählen, Datenbanken-Abfragen zu formulieren und Information zu modellieren.

Ein weiterer Aspekt des «technischen» Aufgabenbereichs ist die Algorithmisierung. Ein Verständnis der Rolle der Technik und ihrer Möglichkeiten in automatisierten und teilautomatisierten Kommunikationsprozessen setzt voraus, dass die Prinzipien maschineller Informationsverarbeitung verstanden sind. Hinzu kommt Orientierungs-Wissen über die technische Infrastruktur, auf die computerbasierte Medien angewiesen sind, etwa zum Aufbau vernetzter Informatiksysteme und Protokolle zum Datenaustausch (ausführlicher in [GI99]). 
Im Unterricht soll auch deutlich werden, dass neben den technischen Einflussfaktoren auch spezifische Interessenslagen einzelner gesellschaftlicher Akteure auf die technischen Grundlagen von Mediengestaltungen einwirken. Ein aktuelles Beispiel sind die Versuche, Kopierschutzmechanismen in Datenformate für Musikdateien zu integrieren (siehe das Beispiel in [GH01]).

\section{Zur Integration der informatischen Aufgabenbereiche in ein Medien- bildungskonzept}

Bei dem Konzept der informatischen Aufgabenbereiche der Medienbildung handelt es sich um einen analytischen Ansatz, der die informatischen Anteile und Voraussetzungen für eine Medienbildung beschreibt.

Behandelt man Ziele des ausdrucksbezogenen Aufgabenbereichs in einem Anwendungsszenario, beispielsweise im Zusammenhang mit der Erstellung einer Schulhomepage, stellen sich sofort Fragen nach dem Verhältnis von Form und Inhalt, von Text und Bild, oder nach der adressatenbezogenen Angemessenheit des zu erstellenden Angebots, zu der die «neutrale» informatische Beschreibungssprache nichts beiträgt. Diese gestalterischen, ästhetischen und inhaltlichen Aspekte werden als fachübergreifende Inhalte in den informatischen Aufgabenbereichen nicht formuliert, da sie in den medienerzieherischen Aufgabenbereichen ausreichend berücksichtigt und in Fächern wie Kunst und Deutsch thematisiert werden.

In der unterrichtlichen Praxis wird man die hier vorgenommene analytische Trennung in informatische und nicht-informatische Aspekte der Medienbildung nicht aufrecht erhalten können - und auch nicht wollen. Die informatischen Aufgabenbereiche sollen, eingebettet in den Informatikunterricht, gegebenenfalls als fachliche Vorbereitung für ein anschliessendes Medienprojekt oder direkt im Zusammenhang mit fächerverbindenden Unterrichtsvorhaben zur Medienbildung berücksichtigt werden. Mit ihrer Hilfe können Anschlussstellen für inhaltliche Angebote zwischen dem Informatikunterricht, anderen Fächern und fächerübergreifenden Medienprojekten deutlicher sichtbar werden.

Wenn etwa im Rahmen des Informatikunterrichts der Sekundarstufe I Inhalte aus dem ausdrucksbezogenen Aufgabenbereich und technischen Grundlagen an Beispielen vermittelt werden, liefern diese die 〈Basis〉, um in spezifischen Medienprojekten Medienangebote angemessen auszuwählen und zu nutzen, zu gestalten und zu verbreiten. Fragen nach Bedeutung und Aufbau der Inhalte, nach den Adressaten des Angebots, nach dem $\mathrm{Zu}$ -
${ }^{3}$ Eliza ist ein Computerprogramm, welches einen Dialog scheinbar verstehend führen kann. Tatsächlich werden die Antworten nach einfachen Regeln und Schlüsselworten generiert. Im Internet findet man Versionen des Programms, die man direkt im Browser ausprobieren kann, zum Beispiel [BS]. (Siehe auch den Beitrag von M. Weigend in dieser Ausgabe.) 
sammenhang zwischen Inhalt und ästhetischer Gestaltung können dann eher ausserhalb des Informatikunterrichts bzw. in Zusammenarbeit mit anderen Fächern eingebracht werden.

Querverbindungen zwischen

informatischer und medienpädagogischer sichtweise

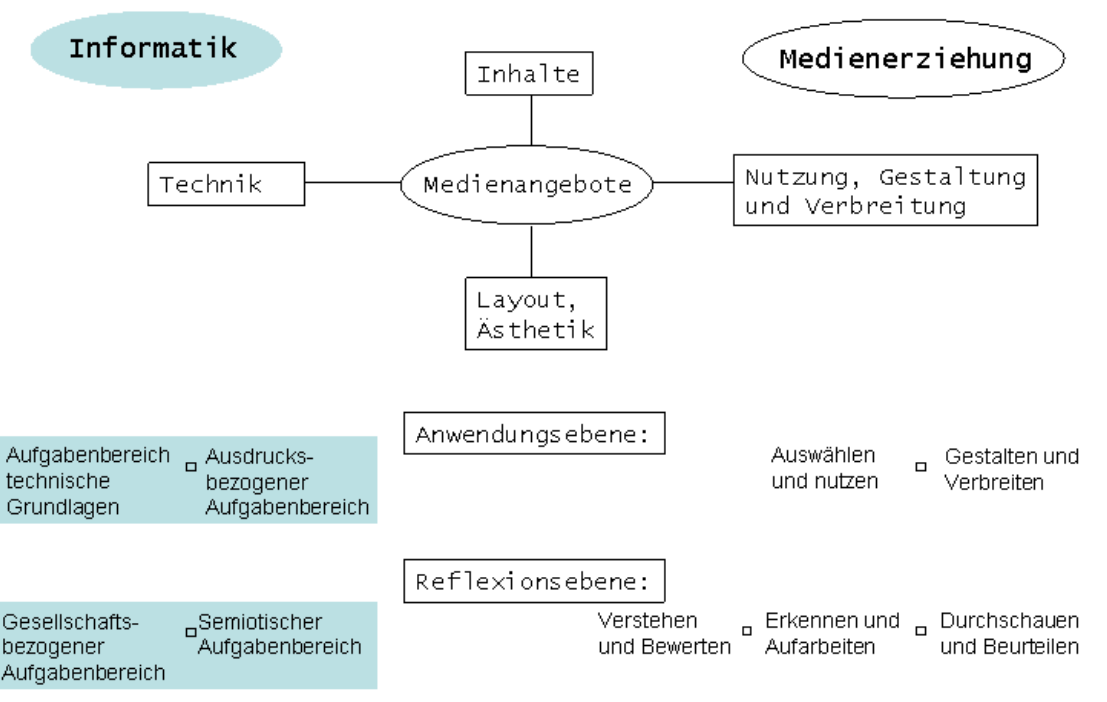

Abb. 3: Verbindungen zwischen informatischen und medienerzieherischen Aufgaben

In solchen handlungsorientierten Medienprojekten sollten dann, ggf. in Zusammenarbeit mit dem Informatikunterricht, auch der dritte und vierte informatische Aufgabenbereich erschlossen werden (siehe das Beispiel aus [Sc01b]). Die Besonderheiten der technischen Grundlagen der Informatik geben oft dazu Anlass, die Wechselwirkungen zwischen Informatik und Gesellschaft sowie die angesprochenen besonderen Rückbezüglichkeiten informationstechnischer Artefakte zu reflektieren bzw. bewusst zu machen. In ähnlicher Weise kann, aufbauend auf dem ausdrucksbezogenen Aufgabenbereich, der semiotische Bereich erschlossen werden, um die spezifischen Möglichkeiten durch automatisierte Zeichenverarbeitungsprozesse $\mathrm{zu}$ verdeutlichen und zu hinterfragen.
Die verschiedenen informatischen und medienerzieherischen Aufgabenbereiche können auf diese Weise recht gut miteinander verzahnt werden. Brichzin und Stolpmann haben ein schulbezogenes Modell eines Medienund Methodencurriculums vorgestellt, in dem u.a. Beiträge der Informatik gezielt mit Inhalten anderer Fächer verknüpft werden: «Es gibt pro Jahrgangsstufe ein Fach, welches einen Themenschwerpunkt des eigenen Fachlehrplans in Form eines Projekts erarbeitet. Andere Fächer stellen unterstützend Module zur Verfügung, d.h. Bausteine, in denen Arbeitstechniken thematisiert und an konkreten Aufgabenstellungen aus dem laufenden Unterricht geübt werden. Diese Module werden im sich zeitlich anschliessenden Projekt genutzt, so dass man sich dort auf Inhalte konzentrieren kann» [BS01].

Mit der Entwicklung ähnlicher übergreifender schulbezogener Konzepte wird die Verbindlichkeit der Medienbildung erhöht: Zum ersten werden die Anteile der einzelnen Fächer an der Medienbildung deutlicher wahrnehmbar, so dass auch im Fachunterricht aus fachlicher Sicht medienbildende Aufgaben etwa im Sinne der hier vorgestellten informatischen Aufgabenbereiche gesehen und auch für die Schülerinnen und Schüler sichtbar gemacht werden. Zum zweiten wird klar, dass Medienbildung eine fächerübergreifende Aufgabe ist, die aber auf die in den Fächern gelegten Grundlagen zurückgreifen kann. Damit muss in einem fächerübergreifenden Projekt eine Lehrkraft nicht alle fachlichen Grundlagen vermitteln, sondern kann auch auf Vorarbeiten anderer aufbauen. 
Literatur

[Br01] Brunnstein, K.: «Mit IT-Risiken umgehen lernen: über Probleme der Beherrschbarkeit komplexer Informatiksysteme.» In: KeilSlawik, R. / Magenheim, J. (Hrsg.): Informatikunterricht und Medienbildung. INFOS 2001. 9. GI-Fachtagung Informatik und Schule. Bonn: Köllen, 2001, S. 9-12

[BS01] Brichzin, P. / Stolpmann, E.: «Medien- und Methodencurriculum Gymnasium Ottobrunn.» In: Keil-Slawik, R. / Magenheim, J. (Hrsg.): Informatikunterricht und Medienbildung. INFOS 2001 9. GI-Fachtagung Informatik und Schule. Bonn: Köllen, 2001, S. $63-73$

[BS] Schwitzer, B.: Eliza. Website mit Eliza-Applet. <www.informatik uni-bremen.de/ deejey/mms/> (besucht am 10.10.01)

[GH01] Himmelein, G.: «Geschenk mit Pferdefuss. Rights Management im Online-Fanclub.» $c / t$ 13/2001. S. 39. Online unter: 〈http://www.heise.de/ct/01/13/039/〉 (besucht am 10.10.01)

[GI99] Gesellschaft für Informatik: Informatische Bildung und Medienerziehung. Empfehlungen der Gesellschaft für Informatik e.V. erarbeitet von einem Arbeitskreis des Fachausschusses 7.3 «Informatische Bildung in Schulen». In: LOG IN 19. Heft 6 , 1999, Beilage

[HB97] Hubwieser, P. / Broy, M.: «Ein neuer Ansatz für den Informatikunterricht am Gymnasium.» In: $\log$ In 17, Heft 3/4, 1997, S. $42-47$

[HT96] Hauf-Tulodziecki, A. / Tulodziecki, G.: «Der Computer als Medium. Medienerzieherische Sichtweisen für die informationstechnische Bildung.» LOG IN 16, Heft 3, 1996, S. 15-22

[Ke00] Keil-Slawik, R.: «Zwischen Vision und Alltagspraxis: Anmerkungen zur Konstruktion und Nutzung typographischer Maschinen.» In: Voss; Holly / Boehnke (Hrsg.): Neue Medien im Alltag: Begriffsbestimmungen eines interdisziplinären Forschungsfeldes. Opladen: Leske \& Budrich, 2000, S. 199-220

[Le80] Lehman, M.: «Programs, Life Cycles, and Laws of Software Evolution.» In: Proceedings of the IEEE, Vol. 68, No. 9, September 1980

[Le99] Lessig, Lawrence: Code and other laws of cyberspace. New York: Basic Books, 1999.

[Ma] Magenheim, J.: «Informatiksystem und Dekonstruktion als didaktische Kategorien - Theoretische Aspekte und unterrichtspraktische Implikationen einer systemorientierten Didaktik der Informatik.»

〈http://ddi.uni-paderborn.de/didaktik/Veroeffentlichungen〉 (besucht am 10.10.01)

[Ma99] Maurer, H.: «WWW needs more interaction.» In: Beiersdörfer / Engels / Schäfer (Hrsg.): Informatik 99. Informatik überwindet Grenzen. Jahrestagung der Gesellschaft für Informatik. Berlin: Springer, 1999

[PSW98] Peschke, R. / Schulz-Zander, R. / Wagner, W.- R.: «Aktive Medienarbeit mit Multimedia. Auf dem Weg zu einer integrierten Medienerziehung.» Computer und Unterricht 29, 1998, S. 5-9

[Sc01a] Schulte, C.: «Vom Modellieren zum Gestalten. Objektorientierung als Impuls für einen neuen Informatikunterricht?» In: informatica didactica. Zeitschrift für fachdidaktische Grundlagen der Informatik, 2001 Ausgabe Nr. 3 http://www. informatica-didactica.de/InformaticaDidactica/〉 (besucht am 10.10.01)

[Sc01b] Schulte, Carsten / Scheel, Olaf: «Aufgabenbereiche der Medienbildung im Informatikunterricht.» In: Keil-Slawik, R. / Magenheim, J. (Hrsg.): Informatikunterricht und Medienbildung. INFOS 2001 9. GI-Fachtagung Informatik und Schule. Bonn: Köllen, 2001, S. 157-171

[Tu01] Tulodziecki, G.: «Medienkompetenz als Ziel schulischer Medienpädagogik.» In: Medienimpulse. Beiträge zur Medienpädagogik. Heft 36, Juni 2001, S. 4-11

[We95] Weingarten, R.: «Das Alphabet in neuen Medien.»In: Osnabrücker Beiträge zur Sprachtheorie (OBST), Bd. 50, 1995, S. 61-82

[Wi00] Wilkens, U.: Das allmähliche Verschwinden der informationstechnischen Grundbildung. Zum Verhältnis von Informatik und Allgemeinbildung. (Diss.) Shaker Verlag, Aachen, 2000, S. 39ff und 130-138 